\title{
Maternal and neonatal outcomes in COVID-19 infected pregnancies: a prospective cohort study
}

Reihaneh Pirjani ${ }^{1}$, Reihaneh Hosseini ${ }^{2}$, Tahereh Soori ${ }^{3}$, Maryam Rabiei ${ }^{2}$, Ladan Hosseini ${ }^{4}$, Amene Abiri ${ }^{1}$, Ashraf Moini ${ }^{5}$, Arshia shizarpour ${ }^{3}$, Ghazal Razani ${ }^{6}$, and Mahdi Sepidarkish ${ }^{7}$

${ }^{1}$ Affiliation not available

${ }^{2}$ Obstetrics and Gynecology Department, Arash Women's Hospital, Tehran University of Medical Sciences, Tehran, Iran

${ }^{3}$ Tehran University of Medical Sciences

${ }^{4}$ Research development center, Arash Women's Hospital, Tehran University of Medical Sciences, Tehran, Iran

${ }^{5} 1$ Department of Endocrinology and Female Infertility, Reproductive Biomedicine Research Center, Royan Institute for Reproductive Biomedicine, ACECR., Tehran, Iran 2Obstetrics and Gynecology Department, Arash Women's Hospital, Tehran University of Medical Sciences, Tehran, Iran.

${ }^{6}$ Arak University of Medical Sciences

${ }^{7}$ Department of Epidemiology and Reproductive Health, Reproductive Epidemiology Research Center, Royan Institute for Reproductive Biomedicine, ACECR, Tehran, Iran.

May 11, 2020

\begin{abstract}
Objective:To compare maternal and fetal consequences of Covid-19-infected pregnancies with non-infected pregnancies. Design: Prospective cohort study Setting: Iran Population: Pregnant women who were hospitalized from March 1 to April 202020 Methods: Participants were divided into three groups: Women with positive Covid-19 PCR test and/or positive CT scan findings in the presence of very typical clinical symptoms were classified as confirmed group,women with typical clinical symptoms along with two or more oflaboratory findings were classified as probable group and women who hadno clinical symptom of infection were classified as a control group.In order to minimize the presence of asymptomatic individuals in the control group, we used very precise exclusion criteria. Main Outcome Measures: Maternal, neonatal and pregnancy outcomes. Results: One hundred and eighty-two women enrolled including37 and 12 and 133 women inconfirmed, probable andcontrol groups respectively.Caesarean Section was carried outin total 96 women $(52.74 \%, 95 \%$ CI:45.22,68.31). Nosignificant association was found between COVID-19 infection and preterm labour(aRR:0.75,95\%CI:0.30,1.87,P=0.546), low birth weight(aRR:1.15,95\%CI:0.49,2.73,P=0.737), gestational diabetes(aRR:0.78,95\%CI:0.26,2.33,P=0.669), preeclampsia (aRR:2.07,95\%CI:0.50,8.47,P=0.310) intrauterine growth restriction(aRR:0.30, 95\%CI:0.02,3.27,P = 0.327) preterm rupture of membrane(aRR:0.38,95\%CI:0.04,3.30,P=0.383), stillbirth (aRR: $1.53,95 \% \mathrm{CI}: 0.15,14.71, \mathrm{P}=0.712$ ), postpartum haemorrhage (aRR:0.80,95\%CI:0.11,5.57,P=0.829), neonatal intensive care unit admission (aRR:1.48,95\%CI:0.38,5.78,P=0.568), neonatal sepsis(aRR:1.23,95\%CI:0.68,2.20,P=0.481) Conclusion:Given the effect of many potential factors, such as the severity of maternal infection, the time interval between the onset of symptoms in mother and childbirth, and many other factors on the effect of the virus on pregnancy outcomes, we suggest conductingmore extensive prospective studies in this field.
\end{abstract}

\section{Introduction}


In recent months and during the rapid outbreak of corona virus disease in 2019 (Covid-19) all around the world, many pregnant women, like other people, have been infected with this deadly virus. Nevertheless, no adequate data is available on the clinical characteristics, manifestations, outcomes, and complications of this virus in pregnancy. Physiological respiratory and non-respiratory changes during pregnancy can predispose pregnant women to COVID-19 infection and aggravate outcomes (1). Some physiological changes in pregnancy include decreased lung volume and functional residual volumes due to diaphragmatic elevations, airway edema, increased oxygen consumption, hyper coagulation state, and altered cell immunity. It seems that these physiological changes can potentially lead to severe pulmonary infectious diseases during pregnancy.In previous pandemics such as H1N1, pregnancy increased susceptibility to serious illnesses and higher mortality rates than the general population (2).A 2009 study found out that women with H1N1 were four times more likely to be hospitalized(3) and also maternal mortality rate from the SARS virus has been reported about 25\% (4) Despite this background, the majority of reported cases of COVID-19 infection in pregnancy have been classified as mild features of the disease and not more severe than general population(5). Most previous studies on the virus during pregnancy have been devised in the form of retrospective studies, case reports and case series. In fact, available evidence on the effects of the virus on pregnant women is still insufficient and inconclusive and there is no consensus between the experts about the virus and pregnancy and also many questions have remained unanswered and need further investigation. With the advent of Covid-19 pandemic, a number of articles have been published about this virus and pregnancy mostly associated with China. At a time that the virus is spreading swiftly around the globe, there is an inevitably urgent need for some well-designed studies in different countries based on diverse nationalities in order to be able to deal with the virus in pregnant women based on strong and solid scientific evidence. Really as far as we know, there has not been any prospective study in this area. so we decided to design a prospective cohort study to compare maternal and fetal consequences of Covid-19-infected pregnancy with non-infected pregnancy.

\section{Method}

This study is a prospective cohort study conducted in Arash women's hospital, a university hospital affiliated with Tehran University of Medical Sciences.

This study has been approved by the Ethics Committee of Tehran University of Medical Sciences (IR.TUMS.VCR.REC.1398.1057).Written consent was obtained from all participants.

In this study, all pregnant women who were hospitalized in Arash Hospital from March 1 to April 202020 were evaluated in terms of inclusion and exclusion criteria. Inclusion criteria were all pregnant women who had been hospitalized in our hospital during the study period and were willing to participate in the study.

Based on the presence or absence of clinical signs or symptoms of covid-19 infection, women were divided into two general categories: One group whose participants had clinical signs and symptoms of the virus and the other group who did not show any clinical signs and symptoms. Clinical symptoms have included fever, dry and progressive cough, tachypnea, shortness of breath, decreased oxygen saturation and fatigue. Reverse transcriptase-polymerase chain reaction for Covid-19 nucleic acid (Covid-19 PCR) test of nasopharyngeal swabs and Chest Computed Tomography (CT) scan were performed for the group with clinical symptoms. Women with positive Covid-19 PCR test were classified as confirmed group. In cases with negative Covid-19 PCR test, if they had very typical clinical symptoms verified by an infectious disease specialist, considering the false negative probability of Covid-19 PCR test and high diagnostic sensitivity of CT scan finding(6, 7), positive findings of CT scan have been considered as a criterion utilized as a tool to confirm the infection. These findings in chest CT scan included ground-glass opacity, consolidation, reticulation/thickened interlobular septa and nodules(6).

Women with negative results of both CT scan and Covid-19 PCR test who had typical clinical symptoms were considered as a potentially infected (probable group) if they had two or more of the laboratory findings including: leukocytosis (white blood cell count $>11 \times 10^{9} / \mathrm{L}$ ), lymphopenia(lymphocyte count $\left.<1.0 \times 10^{9} / \mathrm{L} ;\right)$, thrombocytopenia , increased C-reactive protein $(\mathrm{CRP})([?] 10 \mathrm{mg} / \mathrm{d})$. Women with clinical 
symptoms were excluded from the study in case Covid-19 PCR test and CT scans finding were negative and no laboratory findings were found. Women who did not have any clinical signs or symptom of infection were considered as a control group.

The exclusion criteria for confirmed group were positive CT scan finding in women with mild or transient symptoms in the absence of typical clinical symptoms. The exclusion criteria for probable group were presence of only one paraclinical finding or the presence of only mild and transient clinical symptoms.

In our hospital, universal screening is not performed for all inpatients and since some of affected people may be asymptomatic(8), in order to minimize the presence of asymptomatic individuals in the control group as much as possible, we implemented precise exclusion criteria. So exclusion criteria for control group included: A history of any suspicious symptoms of the virus over the past two weeks, contact with Covid-19 virus infected or suspected individuals within the last 14 days, any suspicious clinical symptoms of the virus over the last two weeks in their family members, attendance in crowded areas such as shopping malls and parties over the past 14 days, going to hospitals or clinics for any reason within the last 14 days except for the time of current hospitalization.

All pregnant women who were hospitalized during the study period were screened according to the above inclusion and exclusion criteria.

Confirmed and probable infected women were treated according to the protocols of the World Health Organization. All other managements and procedures in three groups were performed based on obstetrics indications and our hospital protocols. Given the lack of strong evidence according to perform cesarean section (CS) in women infected by the virus, our hospital's protocol was choosing delivery method based on other obstetrics indications. So we didn't impose CS on women because of corona virus infection. It's worth mentioning that, in our hospital, women give birth in separate rooms during childbirth, and during this epidemic, we have decided to avoid using common tools such as birth balls as much as possible and also doctors, residents, and midwives who take care of each patient are specific for the same patient and do not take care of different patients at the same time as well as use of complete personal protective equipment.

Babies born to infected mothers underwent early cord clamping and early temporary separation from their mothers immediately after birth and Covid-19 PCR test was done via their nasopharyngeal and throat within the same hour after birth.

\section{Statistical analysis}

Continuous variables were described by mean +- standard deviation (SD). Categorical variables were described by counts and percentages (\%).Prenatal and neonatal outcomes of three groups were compared using the Chi-square test for categorical variables, and Student's t-test for continuous variables. We estimated the risk ratios (RRs) and 95\% CIs for categorical adverse pregnancy and neonatal outcomes using Modified poisson regression model. In a few instances, the models did not converge and Firth's bias-reduced penalized-likelihood logistic regression was used. Also A linear mixed effect model was used to analyse changes in continuous adverse pregnancy and neonatal outcomes. The models were adjusted for the following potential confounders: gestational age, parity, body mass index, maternal age, previous caesarean section, previous pregnancy problems, pre-existing medical problems, and mode of delivery. Statistical analyses were performed on Stata16.0 (Stata Corp, College Station, TX, USA). All statistical tests were two-tailed at the significance level of $\mathrm{P}<0.05$.

\section{Results}

During the study period, 436 people were admitted to our hospital with complain of respiratory problems and were evaluated by an infectious disease specialist. Among them, 171 people were suspected of being infected with Covid-19 virus and underwent diagnostic evaluation that 66 of them have been hospitalized and among them, according to inclusion and exclusion criteria, 37 women were included in confirmed group and 12 women were included in probable group.During this time, 652 non-infected women were also hospitalized in 
our hospital which due to some detailed exclusion criteria, only 133 of them entered the study as control group.

\section{Clinical and laboratory findings of infection in confirmed and probable Covid-19 infected preg- nant women}

Thirty four women in confirmed and probable groups were delivered and 15 women continued their pregnancy by the end of study. As illustrated in Table 1, fever (53.06\%, 95\%CI:39.08, 67.03) was the most frequently observed symptom, followed by dry cough $(46.93 \%, 95 \%$ CI:32.96, 60.91$)$, dyspnea $(48.97 \%, 95 \%$ CI: 34.98 , $62.97)$ and myalgia $(25.80 \%, 95 \%$ CI:11.85, 44.61). A total of six $(12.24 \%, 95 \%$ CI: $4.62,24.76)$ patients had decreased oxygen saturation. The most frequent abnormality in laboratory tests included: Raised CRP (63.26\%, 95\% CI: 49.76, 76.76), leukocytosis (white blood cell count >11x10 $/$ L) $(44.89 \%, 95 \%$ CI: 30.97, $58.82)$, and lymphopenia $(32.65 \%, 95 \%$ CI: 19.52, 45.78). There was no difference between confirmed and probable cases in terms of CRP $(\mathrm{p}=0.227)$, leukocytosis $(\mathrm{p}=0.354)$, and lymphopenia $(\mathrm{p}=0.416)$ an as well as other clinical and paraclinical findings(Table 1$)$. Two patients $(4.08 \%, 95 \%$ CI: 0.41, 13.97)were admitted to intensive care unit none of which required intubation and invasive ventilation.

\section{Clinical characteristics and pregnancy outcomes in three groups}

Age range of participants was 18 to 46 years, the mean age was 29.63 years $(\mathrm{SD}=6.89)$ in confirmed and probable groups and 30.89 (6.85) in control group $(\mathrm{P}=0.045)$. Among all participants, $88.3 \%$ of women were in the third trimester of pregnancy that $82.9 \%$ and $58.3 \%$ and $92.4 \%$ of them were in confirmed, probable and control groups respectively. Interestingly, probable grouphad lower gestational age [30.09 (11.01)] in comparison with confirmed [33.43 (7.50) and control groups [36.57 (5.30)] $(\mathrm{P}<0.001)$. There were no differences between these groups regarding clinical characteristics and past medical and obstetric history (Table 2). Furthermore, confirmed cases had almost the same frequency of pre-existing medical problems (13.51\%) compared to probable cases $(8.33 \%)$ and control group $(12.03 \%)(\mathrm{p}=0.688)$. It should be noted that since hypothyroidism was common in all three groups, especially in the control group, we did not classify hypothyroidism with other underlying diseases in the same category to prevent its confounding effect.

The unadjusted and adjusted estimates for total maternal and neonatal outcomes are presented in Table 3. CS was carried out in total 96 women $(52.74 \%, 95 \%$ CI: 45.22, 68.31). Although unadjusted results showed significantly higher rate of CS in probable group compared with confirmed and control groups, after adjusting for potential confounders, no significant differences were found in terms of delivery type between confirmed and probable groups (aRR: 1.24, 95\%CI: $0.93,1.65, \mathrm{P}=0.138$ ) and also between probable and control groups (aRR: $1.55,95 \%$ CI: $0.85,2.82, \mathrm{P}=0.152$ ), so, there was no difference between groups according to delivery type. In addition, no significant association was found between COVID-19 infection and preterm labor(PTL), gestational diabetes mellitus (GDM), preeclampsia, intra uterine growth restriction(IUGR),preterm rupture of membrane (PROM),stillbirth, postpartum haemorrhage and postpartum infection. Furthermore, there were no significant differences between three groups in terms of essential neonatal outcomes including low birth weight, NICU admission, neonatal sepsis, APGAR score at one and 5 minutes (Tables 3 and 4).In our study, only one infant had a positive Covid-19 PCR test after birth, and the other infants had negative results. Considering this issue that the baby was completely isolated and separated from the mother immediately after birth, there is a possibility of vertical transmission in this baby, but anyway, since we did not examine the umbilical cord blood and amniotic fluid in terms of virus culture, we cannot definitively claim that there has been a vertical transmission in this baby

\section{Discussion}

\section{Main finding}

In this prospective cohort study we compared pregnancy in women infected by Covid-19 virus with noninfected pregnant women. Fever, dry cough, dyspnea and myalgia were the most frequently observed symptom in infected women. Overall, $88.3 \%$ of participants were in the third trimester of pregnancy. There were no differences between groups according to delivery type and also maternal and neonatal outcomes. In 
addition, no significant association was found between COVID-19 infection and adverse pregnancy outcomes.

\section{Strengths and limitations}

One of the strengths of our study is that it was designed as a prospective cohort study, while most of the previous studies were case reports and series and a few studies had a retrospective case-control design. The other strength of our study was that we didn't consider only women who had positive COVID-19 PCR test as a confirmed group, but given the probability of false negative results in the virus PCR, those who had positive CT scan findings in the presence of very typical clinical symptoms were also considered as confirmed infected people, so in this way, we tried not to overlook the infected women as much as possible.As we explained in method section, one of the strengths of our study was choosing very precise exclusion criteria for all three groups, especially for the control group.

One of the limitations of our study is that there is no universal screening for all pregnant women in our hospital, so it is highly likely for some asymptomatic infected women to be found among non -infected group.To overcome this limitation, we have made our best effort to select non-infected women very meticulously as we described in method section. Another limitation of the study, however, was the short duration of the study. Although the sample size of our study is higher than most of previous studies on covid-19 and pregnancy, we believed that If we continued to study, we could increase the sample size, but given the current outbreak of the virus worldwide, we prefered to present our data as soon as possible in order to amass accurate data in this field.

\section{Interpretation}

In this study, we have tried to address some issues including: the severity of Covid-19 virus infection in pregnancy, the risk of virus infection with pregnancy progress, the virus impact on pregnancy outcomes, delivery type, the possibility of vertical transmission and neonatal consequences.

Studies to date have shown that Covid-19 virus infection is not more severe in pregnant women.(9-12). We also did not have any severe case of corona virus during our study.However, it should be noted that our study was performed only during 50 days and therefore, the absence of a critically ill patient during this period cannot be generally attributed to all pregnancies. In late February 2020, a very ill 22-year-old pregnant woman with no underlying disease who had been infected by Covid-19 virus was referred to our hospital at 37 gestational weeks due of fever and severe shortness of breathing that had started ten days earlier. She developed lymphopenia, thrombocytopenia and low O2 saturation (60\%) and unfortunately passed away within 7 hours of admission. Since this happened before beginning of our cohort study, we did not include her in this study.Recently, Hantoushzadeet al. has reported nine pregnant women with severe Covid-19 disease which 7 of them died(13). Their study is a multidisciplinary study that has selected highly ill patients from different centers across Iran, alongside non-random selection bias. However, since no cases of Covid-19 death have been reported in pregnancy so far, their study could make an important change in available evidence about the manifestation of the virus in pregnancy.

Most of the Covid-19 infected pregnant women that have been reported so far were in the third and late second trimesters. $(10,14,15)$.In our study, $88.3 \%$ of those surveyed were in the third trimester of pregnancy. Really, the possibility of picking up infected women based on careful examination and history at earlier ages of pregnancy is lower because of the less routine prenatal visits in first and second trimesters. On the other hand, given the general policies around the world regarding home quarantine during a virus pandemic, pregnant women may not go to medical centers even if there are mild symptoms. So we believe that previous reports including our study might be influenced by these factors and the higher average gestational age in our patients should be interpreted with caution.

In our study, there was no increase in adverse pregnancy outcomes including PTL,GDM, preeclampsia, IUGR, PROM, stillbirth, postpartum haemorrhage and postpartum infection. Most previous studies including a review article(16)also had the same results. Since in most previous studies no comparison has been made between infected and non-infected individuals duo to their methodology, it is not possible to give a definite 
opinion about the effect of this virus on pregnancy outcomes based on the available data and it demands more prospective studies.

Our results showed that CS rate was not different in the studied groups, but in the majority of previous studies, it was rather higher in infected people(10, 14, 16-18). In a review study, fetal distress was reported to be the most common cause of CS in infected women(17).However, In our study, the most common cause of CS was a history of previous CS.

In our study, only one infant had a positive Covid-19 PCR test after birth that it cannot be definitively related to vertical transmission. Although no decisive vertical transmission of the virus has been reported so far,there area few reports of covid-19 viral pneumonia in 3 infants(19),elevated SARS-Cov-2 IgM and IgG levels in 2 hours after birth in a newborn(20)and a newborn whose nasopharyngeal culture was positive for covid-19 virus 16 hours after $\operatorname{birth}(21)$.

Since several factors may play some roles in the vertical transmission, we recommend culture of amniotic fluid, umbilical cord blood and neonatal nasopharyngeal swab at the time of delivery in order to accurately determine the vertical transmission.

Several studies have reported neonatal complications in some of their neonates(10, 19, 22), whereas in some other studies, no neonatal complication has been observed $(14,23)$. The prevalence of neonatal complications was not different between groups in our study. Nevertheless, we have the impression that maternal condition during peak of the disease may lead to severe transient placental insufficiency. We suggest evaluating uterine and umbilical vessels doppler at the onset and peak of the disease and also after the mother's recovery and comparing them with neonatal outcomes in futures studies.

\section{Conclusion}

No differences were found between Covid-19 infected and non-infected pregnant women according to delivery type, maternal and neonatal outcomes and also adverse pregnancy outcomes. Unfortunately, there is still no enough evidence about Covid-19 virus in pregnancy. Given the effect of many potential factors, such as the severity of maternal infection, the time interval between the onset of symptoms in mother and childbirth, the adequacy of the placental blood supply and many other factors on the effect of the virus on pregnancy outcomes, we suggest conducting more extensive prospective studies in this field. Future studies could lead to changes in current knowledge.

Disclosure of interests: The authors did not report any potential conflicts of interest

Contribution to authorship: Study design and protocol development: RP,MS , Data analysis: M S, Subject recruitment and follow up: RH,RP, TS,GR,ASH,MR,AR,AM. Manuscript preparation: RP,MS Manuscript review and submission: RP,MS,LH. All authors read and approved the final manuscript

Details of ethics approval: This study was approved by the Institutional Review Board and the Ethics Committee of Tehran University of Medical Sciences, Tehran, Iran. (IR.TUMS.VCR.REC.1398.1057) and all participants submitted written informed consent.

Funding: This research has been supported by a research grant

No: 99-1-101-47129from Deputy of Research affairs, Tehran University of Medical Sciences, Tehran ,Iran.

\section{References}

1. Dashraath P, Jeslyn WJL, Karen LMX, Min LL, Sarah L, Biswas A, et al. Coronavirus disease 2019 (COVID-19) pandemic and pregnancy. American Journal of Obstetrics and Gynecology. 2020.

2. Rasmussen SA, Jamieson DJ, Bresee JS. Pandemic influenza and pregnant women. Emerging infectious diseases. 2008;14(1):95.

3. Jamieson DJ, Honein MA, Rasmussen SA, Williams JL, Swerdlow DL, Biggerstaff MS, et al. H1N1 2009 influenza virus infection during pregnancy in the USA. The Lancet. 2009;374(9688):451-8. 
4. Wong SF, Chow KM, Leung TN, Ng WF, Ng TK, Shek CC, et al. Pregnancy and perinatal outcomes of women with severe acute respiratory syndrome. American journal of obstetrics and gynecology. 2004;191(1):292-7.

5. Qiao J. What are the risks of COVID-19 infection in pregnant women? The Lancet. 2020;395(10226):7602 .

6. Ai T, Yang Z, Hou H, Zhan C, Chen C, Lv W, et al. Correlation of chest CT and RT-PCR testing in coronavirus disease 2019 (COVID-19) in China: a report of 1014 cases. Radiology. 2020:200642.

7. Long C, Xu H, Shen Q, Zhang X, Fan B, Wang C, et al. Diagnosis of the Coronavirus disease (COVID-19): rRT-PCR or CT? European journal of radiology. 2020:108961.

8. Breslin N, Baptiste C, Gyamfi-Bannerman C, Miller R, Martinez R, Bernstein K, et al. COVID-19 infection among asymptomatic and symptomatic pregnant women: Two weeks of confirmed presentations to an affiliated pair of New York City hospitals. American Journal of Obstetrics \& Gynecology MFM. 2020:100118.

9. Liu D, Li L, Wu X, Zheng D, Wang J, Yang L, et al. Pregnancy and perinatal outcomes of women with coronavirus disease (COVID-19) pneumonia: a preliminary analysis. American Journal of Roentgenology. 2020:1-6.

10. Chen H, Guo J, Wang C, Luo F, Yu X, Zhang W, et al. Clinical characteristics and intrauterine vertical transmission potential of COVID-19 infection in nine pregnant women: a retrospective review of medical records. The Lancet. 2020;395(10226):809-15.

11. Huang C, Wang Y, Li X, Ren L, Zhao J, Hu Y, et al. Clinical features of patients infected with 2019 novel coronavirus in Wuhan, China. The lancet. 2020;395(10223):497-506.

12. Li Q, Guan X, Wu P, Wang X, Zhou L, Tong Y, et al. Early transmission dynamics in Wuhan, China, of novel coronavirus-infected pneumonia. New England Journal of Medicine. 2020.

13. Hantoushzadeh S, Shamshirsaz AA, Aleyasin A, Seferovic MD, Aski SK, Arian SE, et al. Maternal Death Due to COVID-19 Disease. American Journal of Obstetrics and Gynecology. 2020.

14. Li N, Han L, Peng M, Lv Y, Ouyang Y, Liu K, et al. Maternal and neonatal outcomes of pregnant women with COVID-19 pneumonia: a case-control study. Clinical Infectious Diseases. 2020.

15. Fan C, Lei D, Fang C, Li C, Wang M, Liu Y, et al. Perinatal Transmission of COVID-19 Associated SARS-CoV-2: Should We Worry? Clinical Infectious Diseases. 2020.

16. Mimouni F, Lakshminrusimha S, Pearlman SA, Raju T, Gallagher PG, Mendlovic J. Perinatal aspects on the covid-19 pandemic: a practical resource for perinatal-neonatal specialists. Journal of Perinatology. 2020:1-7.

17. Zaigham M, Andersson O. Maternal and Perinatal Outcomes with COVID-19: a systematic review of 108 pregnancies. Acta obstetricia et gynecologica Scandinavica. 2020.

18. Yang H, Wang C, Poon L. Novel coronavirus infection and pregnancy. Ultrasound in Obstetrics \& Gynecology. 2020;55(4):435.

19. Zeng L, Xia S, Yuan W, Yan K, Xiao F, Shao J, et al. Neonatal early-onset infection with SARS-CoV-2 in 33 neonates born to mothers with COVID-19 in Wuhan, China. JAMA pediatrics. 2020.

20. Dong L, Tian J, He S, Zhu C, Wang J, Liu C, et al. Possible vertical transmission of SARS-CoV-2 from an infected mother to her newborn. Jama. 2020.

21. Alzamora MC, Paredes T, Caceres D, Webb CM, Valdez LM, La Rosa M. Severe COVID-19 during Pregnancy and Possible Vertical Transmission. American Journal of Perinatology. 2020. 
22. Schwartz DA. An analysis of 38 pregnant women with COVID-19, their newborn infants, and maternalfetal transmission of SARS-CoV-2: maternal coronavirus infections and pregnancy outcomes. Archives of pathology \& laboratory medicine. 2020.

23. Yu N, Li W, Kang Q, Xiong Z, Wang S, Lin X, et al. Clinical features and obstetric and neonatal outcomes of pregnant patients with COVID-19 in Wuhan, China: a retrospective, single-centre, descriptive study. The Lancet Infectious Diseases. 2020.

\section{Hosted file}

Tables.docx available at https://authorea.com/users/320436/articles/449999-maternal-andneonatal-outcomes-in-covid-19-infected-pregnancies-a-prospective-cohort-study 